\title{
General Perspective on Distributionally Learnable Classes
}

\author{
Ryo Yoshinaka \\ Kyoto University, Japan \\ ryei.kyoto-u.ac.jp
}

\begin{abstract}
Several algorithms have been proposed to learn different subclasses of context-free grammars based on the idea generically called distributional learning. Those techniques have been applied to many formalisms richer than context-free grammars like multiple context-free grammars, simple contextfree tree grammars and others. The learning algorithms for those different formalisms are actually quite similar to each other. We in this paper give a uniform view on those algorithms.
\end{abstract}

\section{Introduction}

Approaches based on the idea generically called distributional learning have been making great success in the algorithmic learning of various subclasses of context-free grammars (CFGs) (Clark, 2010c; Yoshinaka, 2012). Those techniques are applied to richer formalisms as well. The formalisms studied so far include multiple CFGs (Yoshinaka, 2011a), simple context-free tree grammars (CFTGs) (Kasprzik and Yoshinaka, 2011), second-order abstract categorial grammars (Yoshinaka and Kanazawa, 2011), parallel multiple CFGs (Clark and Yoshinaka, 2014), conjunctive grammars (Yoshinaka, 2015) and others. The goal of this paper is to present a uniform view on those algorithms.

Every grammar formalism for which distributional learning techniques have been proposed so far generate their languages through context-free derivation trees, whose nodes are labeled by production rules. The formalism and grammar rules deter- mine how a context-free derivation tree $\tau$ is mapped to a derived object $\tilde{\tau}=d$. A context-free derivation tree $\tau$ can be decomposed into a subtree $\sigma$ and a tree-context $\chi$ so that $\tau=\chi[\sigma]$. The subtree determines a substructure $s=\tilde{\sigma}$ of $d$ and the tree-context determines a contextual structure $c=\tilde{\chi}$ in which the substructure is plugged to form the derived object $d=c \odot s$, where we represent the plugging operation by $\odot$. In the $\mathrm{CFG}$ case, $c$ is a string pair $\langle l, r\rangle$ and $s$ is a string $u$ and $\langle l, r\rangle \odot u=l u r$, which may correspond to a derivation $I \stackrel{*}{\Rightarrow} l X r \stackrel{*}{\Rightarrow} l u r$ where $I$ is the initial symbol and $X$ is a nonterminal symbol. In richer formalisms those substructures and contexts may have richer structures, like tuples of strings or $\lambda$-terms. A learner does not know how a given example $d$ is derived by a hidden grammar behind the observed examples. A learner based on distributional learning simply tries all the possible decompositions of a positive example into arbitrary two parts $c^{\prime}$ and $s^{\prime}$ such that $d=c^{\prime} \odot s^{\prime}$ where some grammar may derive $d$ thorough a derivation tree $\tau^{\prime}=\chi^{\prime}\left[\sigma^{\prime}\right]$ with $\tilde{\chi}^{\prime}=c^{\prime}$ and $\tilde{\sigma}^{\prime}=s^{\prime}$. Based on observation on the relation between substructures and contexts collected from given examples, a hypothesis grammar is computed. We call properties on grammars with which distributional learning approaches work distributional properties.

This paper first formally defines grammar formalisms based on context-free derivation trees. We then show that grammars with different distributional properties are learnable by standard distributional learning techniques if the formalism satisfies some conditions, which include polynomialtime decomposability of objects into contexts and 
substructures. In addition, we discuss cases where we cannot enumerate all of the possible contexts and substructures.

\section{$2 \quad \sum$-grammars}

There is a number of ways to represent a language, a subset of an object set $\mathbb{O}_{*}$, whose elements are typically strings, trees but anythings encodable are eligible. Formalisms this paper discusses generate objects in $\mathbb{O}_{*}$ through context-free derivation trees $\tau$, which are mapped to an element $d \in \mathbb{O}_{*}$ in a uniform way. The map is inductively defined and computed. Each derivation subtree $\tau^{\prime}$ of $\tau$ also determines an object, which we call a substructure of $d$. Each substructure is not necessarily a member of $\mathbb{O}_{*}$. For example, nonterminal symbols of multiple CFGs (Seki et al., 1991) derive $n$-tuples of strings, where the value $n$ is unique to each nonterminal, while the languages generated by multiple CFGs are still simply string sets. A generalization of the CFG formalism is specified by kinds of objects that each nonterminal generates and admissible operations over those objects.

Let $(\mathbb{O}$ be a set of objects, which are identified with their codes of finite length. We have a set $\Omega$ of finite representations $O$ which are interpreted as subsets $\mathbb{O}_{O}$ of $\mathbb{O}$ through an effective procedure. By a sort we flexibly refer to $O \in \Omega$ or $\mathbb{O}_{O} \subseteq \mathbb{O}$. We also have an indexed family of computable functions from tuples of objects of some sorts to objects of some sort. Let $\mathbb{F}$ be a set of function names or function indices $f$, which represent functions $\tilde{f}$. By $\mathbb{O}_{1} \times \cdots \times \mathbb{O}_{n} \rightarrow \mathbb{O}_{0}$ we denote the set of functions whose domain is $\mathbb{O}_{1} \times \cdots \times \mathbb{O}_{n}$ and codomain is $\mathbb{O}_{0}$. By $\mathbb{F}_{O_{0}, O_{1}, \ldots, O_{n}}$, we denote the set of function names $f \in \mathbb{F}$ with $\tilde{f} \in \mathbb{O}_{O_{1}} \times \cdots \times \mathbb{O}_{O_{n}} \rightarrow \mathbb{O}_{O_{0}}$. We assume that the domain sorts $O_{1}, \ldots, O_{n}$ and the codomain sort $O_{0}$ are easily computed from $f$. We specify a class of grammars by a triple, which we call a signature, $\Sigma=\left\langle\Omega, \mathbb{F}, O_{*}\right\rangle$ where $O_{*} \in \Omega$ is a special sort of objects. We write $\mathbb{O}_{*}$ for $\mathbb{O}_{O_{*}}$.

A context-free $\Sigma$-grammar ( $\Sigma$-grammar for short) is a tuple $G=\langle N, \sigma, F, P, I\rangle$ where $N$ is a finite set of nonterminal symbols, $I \subseteq N$ is a set of initial symbols, $\sigma \in N \rightarrow \Omega$ is a sort assignment on nonterminals such that $\sigma(X)=O_{*}$ for all $X \in I$, $F \subseteq \mathbb{F}$ is a finite set of function names, and $P$ is a finite set of production rules, which are elements of $N \times F \times N^{*}$. Each production rule is denoted as

$$
X_{0} \leftarrow f\left\langle X_{1}, \ldots, X_{n}\right\rangle
$$

where $X_{0}, \ldots, X_{n} \in N$ and $f \in \mathbb{F}_{O_{0}, O_{1}, \ldots, O_{n}}$ for $\sigma\left(X_{i}\right)=O_{i}$. For each $O \in \Omega, N_{O}=\sigma^{-1}(O) \subseteq N$ is the set of $O$-nonterminals which are assigned the sort $O$. By $\mathcal{G}(\Sigma)$ we denote the class of $\Sigma$ grammars.

A $\Sigma$-grammar defines its language via derivation trees, which are recursively defined as follows.

- If $\tau_{i}$ are $X_{i}$-derivation trees for $i=1, \ldots, n$ and $\rho$ is a rule of the form $X_{0} \leftarrow f\left\langle X_{1}, \ldots, X_{n}\right\rangle$, then the term $\tau_{0}=\rho\left[\tau_{1}, \ldots, \tau_{n}\right]$ is an $X_{0^{-}}$ derivation tree. Its yield $\tilde{\tau}_{0}$ is $\tilde{f}\left(\tilde{\tau}_{1}, \ldots, \tilde{\tau}_{n}\right) \in$ $\mathbb{O}_{\sigma\left(X_{0}\right)}$ where $\tilde{\tau}_{i}$ is the yield of $\tau_{i}$.

The case where $n=0$ gives the base of this recursive definition. An $X$-derivation tree is complete if $X \in I$. The yield of any $X$-derivation tree is called an $X$-substructure. By $\mathcal{S}(G, X)$ we denote the set of $X$-substructures. The language of $G$ is $\mathcal{L}(G)=\bigcup_{X \in I} \mathcal{S}(G, X)$, which we call a $\Sigma$ language. In other words, $\mathcal{L}(G)$ is the set of the yields of complete derivation trees. The class of $\Sigma$ languages is denoted by $\mathcal{L}(\Sigma)$.

Distributional learning is concerned with what $X$ derivation contexts represent. An $X$-derivation context is obtained by replacing an occurrence of an $X$-derivation tree in a complete derivation tree by a special symbol $\square_{\sigma(X)}$. Accordingly the yield $\tilde{\chi}$ of an $X$-derivation context $\chi$ should be a finite representation of a function that gives $\widetilde{\chi[\tau]}$ when applied to $\tilde{\tau}$ for any $X$-derivation tree $\tau$. We assume to have a set $\mathbb{E}_{O}$ of representations of functions from $\mathbb{O}_{O}$ to $\mathbb{O}_{*}$ for $O \in \Omega$ to which the yields of derivation contexts belong.

- $\square_{X}$ is an $X$-derivation context for all $X \in I$ and its yield $\square_{O_{*}} \in \mathbb{E}_{O_{*}}$ represents the identity function on $\mathbb{O}_{*}$,

- For an $X$-derivation context $\chi_{0}$, a rule $\rho=$ $X \leftarrow f\left\langle X_{1}, \ldots, X_{n}\right\rangle$ and $X_{i}$-derivation trees $\tau_{i}$ for $i \in\{1, \ldots, n\}-\{j\}$, the term $\chi$ obtained by replacing $\square_{X}$ in $\chi_{0}$ by $\rho\left[\tau_{1}, \ldots, \tau_{j-1}, \square_{X_{j}}, \tau_{j+1}, \ldots, \tau_{n}\right]$ is an $X_{j}$ derivation context. Its yield $\tilde{\chi} \in \mathbb{E}_{\sigma\left(X_{j}\right)}$, which 
is denoted as

$$
\tilde{\chi}=\tilde{\chi_{0}} \odot \tilde{f}\left(\tilde{\tau}_{1}, \ldots, \tilde{\tau}_{j-1}, \square_{\sigma\left(X_{j}\right)}, \tilde{\tau}_{j-1}, \ldots, \tilde{\tau}_{n}\right),
$$

represents the function $\phi \in \mathbb{O}_{\sigma\left(X_{j}\right)} \rightarrow \mathbb{O}_{*}$ such that for all $s \in \mathbb{O}_{\sigma\left(X_{j}\right)}$,

$$
\phi(s)=\phi_{0}\left(\tilde{f}\left(\tilde{\tau}_{1}, \ldots, \tilde{\tau}_{j-1}, s, \tilde{\tau}_{j+1}, \ldots, \tilde{\tau}_{n}\right)\right),
$$

where $\phi_{0}$ is the function represented by $\tilde{\chi}_{0}$.

The yield of any $X$-derivation context is called an $X$-context. By $\mathcal{C}(G, X)$ we denote the set of $X$ contexts. For $c \in \mathcal{C}(G, X)$ and $s \in \mathcal{S}(G, X), c \odot s$ is the result of the application of the function represented by $c$ to $s$.

\section{Context-substructure relation}

By $\mathbb{S}$ and $\mathbb{C}$ we denote the set of substructures and contexts, respectively, which can be obtained by some grammar in $\mathcal{G}(\Sigma)$ :

$$
\begin{aligned}
& \mathbb{S}=\bigcup_{O \in \Omega} \mathbb{S}_{O} \text { and } \mathbb{C}=\bigcup_{O \in \Omega} \mathbb{C}_{O} \text { where } \\
& \mathbb{S}_{O}=\bigcup\{\mathcal{S}(G, X) \mid X \text { is an } O \text {-nonterminal } \\
& \text { of some } G \in \mathcal{G}(\Sigma) \text { \} } \\
& \mathbb{C}_{O}=\bigcup\{\mathcal{C}(G, X) \mid X \text { is an } O \text {-nonterminal } \\
& \text { of some } G \in \mathcal{G}(\Sigma)\} \text {. }
\end{aligned}
$$

We write $\mathbb{S}_{*}$ for $\mathbb{S}_{O_{*}}$. Note that the above definition is relative to $\Sigma$. Even if $\mathbb{O}_{O_{1}}=\mathbb{O}_{O_{2}}$ for different $O_{1}, O_{2} \in \Omega$, it can be the case that $\mathbb{S}_{O_{1}} \neq \mathbb{S}_{O_{2}}$ and $\mathbb{C}_{O_{1}} \neq \mathbb{C}_{O_{2}}$. Though usually $\mathbb{O}_{O}$ has a definition independent from $\Sigma$, it is possible to specify $\mathbb{O}_{O}$ in terms of the signature so that $\mathbb{S}_{O}=\mathbb{O}_{O}$. Clearly if $s \in \mathbb{S}_{O}$ and $c \in \mathbb{C}_{O}$, then there is a grammar $G \in$ $\mathcal{G}(\Sigma)$ generating $c \odot s$ using a nonterminal of sort $O$. Therefore, $c \odot s$ is well defined for any $s \in \mathbb{S}_{O}$ and $c \in \mathbb{C}_{O}$ without specifying a particular $\Sigma$-grammar. Similarly $c \odot \tilde{f}\left(s_{1}, \ldots, s_{j-1}, \square_{O_{j}}, s_{j+1}, s_{n}\right)$ is well defined for any $c \in \mathbb{C}_{O_{0}}, f \in \mathbb{F}_{O_{0}, \ldots, O_{n}}$ and $s_{i} \in$ $\mathbb{S}_{O_{i}}$. This operation is generalized to sets $S \subseteq \mathbb{S}_{O}$ and $C \subseteq \mathbb{C}_{O}$ in the straightforward way, like $C \odot$ $S=\{c \odot s \mid c \in C$ and $s \in S\}$.

Hereafter, whenever we write $c \odot s$ and $\tilde{f}\left(s_{1}, \ldots, s_{n}\right)$, we assume they are well-formed. That is, the domains of the functions represented by $c$ and $f$ match the sorts to which $s$ and $s_{1}, \ldots, s_{n}$ belong, respectively. Accordingly we drop the subscript $O$ from $\square_{O}$ and write $\tilde{f}\left(s_{1}, \ldots, s_{j-1}, \square, s_{j+1}, \ldots, s_{n}\right)$. When we have a substructure set $S$, we assume $S \subseteq \mathbb{S}_{O}$ for some $O \in \Omega$. We often identify $s$ with $\{s\}$ unless confusion arises. Also we assume $\mathbb{S}_{O} \neq \varnothing$ for all $O \in \Omega$. The same assumptions apply to contexts.

We are interested in whether the composition $c \odot s$ belongs to a concerned language $L \in \mathcal{L}(\Sigma)$. Clark (2010b) has introduced syntactic concept lattices to analyze the context-substring relation on string languages and particularly to design a distributional learning algorithm for CFLs. Generalizing his discussion, we define an $O$-concept lattice $\mathfrak{B}_{O}(L)$ of a language $L \subseteq O_{*}$ for respective sorts $O \in \Omega$. Assuming $L$ and $O$ understood from the context, let us write

$$
\begin{aligned}
& S^{\ddagger}=\left\{c \in \mathbb{C}_{O} \mid c \odot S \subseteq L\right\}, \\
& C^{\dagger}=\left\{s \in \mathbb{S}_{O} \mid C \odot s \subseteq L\right\}
\end{aligned}
$$

for $S \subseteq \mathbb{S}_{O}$ and $C \subseteq \mathbb{C}_{O}$. We write $S^{\dagger}$ for $S^{\ddagger}$ and $C^{\ddagger}$ for $C^{\dagger^{\ddagger}}$.

We call a pair $\langle S, C\rangle \subseteq \mathbb{S}_{O} \times \mathbb{C}_{O}$ a concept iff $S^{\dagger}=C$ and $C^{\ddagger}=S$. For any $S \subseteq \mathbb{S}_{O}$ and $C \subseteq$ $\mathbb{C}_{O},\left\langle S^{\dagger}, S^{\ddagger}\right\rangle$ and $\left\langle C^{\dagger}, C^{\ddagger}\right\rangle$ are concepts. We call them the concepts induced by $S$ and $C$, respectively. For two concepts $\left\langle S_{1}, C_{1}\right\rangle$ and $\left\langle S_{2}, C_{2}\right\rangle$ in $\mathfrak{B}_{O}(L)$, we write $\left\langle S_{1}, C_{1}\right\rangle \leq_{L}^{O}\left\langle S_{2}, C_{2}\right\rangle$ if $S_{1} \subseteq S_{2}$, which is equivalent to $C_{2} \subseteq C_{1}$. With this partial order, $\mathfrak{B}_{O}(L)$ is a complete lattice.

We can introduce a partial order to substructure sets based on the concepts that they induce. Let us write $S_{1} \leq_{L}^{O} S_{2}$ if $S_{2}^{\ddagger} \subseteq S_{1}^{\ddagger}$. The relation represents the substitutability of $S_{1}$ for $S_{2}$.

Lemma 1. The following three are equivalent for $S, T \subseteq \mathbb{S}_{O}$ :

- $S \leq_{L}^{O} T$,

- $c \odot T \subseteq L$ implies $c \odot S \subseteq L$ for all $c \in \mathbb{C}_{O}$,

- $T^{\ddagger} \odot S \subseteq L$.

If $S_{i} \leq_{L}^{O_{i}} T_{i}$ for $i=1, \ldots, n$, then for any $f \in \mathbb{F}_{O_{0}, O_{1}, \ldots, O_{n}}$, we have $f\left(S_{1}, \ldots, S_{n}\right) \leq_{L}^{O_{0}}$ $f\left(T_{1}, \ldots, T_{n}\right)$.

If $S_{1} \leq_{L} S_{2}$ and $S_{2} \leq_{L} S_{1}$, we write $S_{1} \equiv_{L} S_{2}$. 


\section{Conditions to be distributionally learnable}

Distributional learning algorithms decompose examples $d \in \mathbb{S}_{*}$ into contexts $c \in \mathbb{C}_{O}$ and substructures $s \in \mathbb{S}_{O}$ so that $c \odot s=d$. Then a primal approach uses substructures or sets of substructures as nonterminals of a conjecture grammar. We want each nonterminal $\llbracket S \rrbracket$ indexed by $S \subseteq \mathbb{S}_{O}$ to satisfy $\mathcal{S}(G, \llbracket S \rrbracket)=S^{\dagger}$. On the other hand, a dual approach uses contexts or sets of contexts as nonterminals where the semantics of the nonterminal is $\mathcal{S}(G, \llbracket C \rrbracket)=C^{\dagger}$. For an object $d \in \mathbb{S}_{*}, O \in \Omega$ and $\vec{O}=\left(O_{0}, \ldots, O_{n}\right)$ with $O_{i} \in \Omega$, we define

$$
\begin{aligned}
\mathbb{S}_{O \mid d}= & \left\{s \in \mathbb{S}_{O} \mid c \odot s=d \text { for some } c \in \mathbb{C}_{O}\right\} \\
\mathbb{C}_{O \mid d}= & \left\{c \in \mathbb{C}_{O} \mid c \odot s=d \text { for some } s \in \mathbb{S}_{O}\right\} \\
\mathbb{F}_{\vec{O} \mid d}=\left\{f \in \mathbb{F}_{\vec{O}} \mid c \odot f\left(s_{1}, \ldots, s_{m}\right)=d\right. & \left.\quad \text { for some } c \in \mathbb{C}_{O_{0} \mid d} \text { and } s_{i} \in \mathbb{S}_{O_{i} \mid d}\right\},
\end{aligned}
$$

$\mathbb{S}_{\mid D}=\bigcup_{O \in \Omega}^{d \in D} \mathbb{S}_{O \mid d}, \mathbb{C}_{O \mid D}=\bigcup_{O \in \Omega}^{d \in D} \mathbb{C}_{O \mid d}$ and $\mathbb{F}_{\mid D}=$ $\bigcup_{\vec{O} \in \Omega^{*}}^{d \in D} \mathbb{F}_{\vec{O} \mid d}$ for $D \subseteq O_{*}$. Let $\Omega_{\mid D}=\bigcup_{d \in D} \Omega_{\mid d}$ for $\Omega_{\mid d}=\left\{O \mid \mathbb{S}_{O \mid d} \neq \varnothing\right\}$.

We require $\mathcal{G}(\Sigma)$ to be a tractable formalism such that composition and decomposition can be done efficiently.

Assumption 1. There are polynomial-time algorithms which

- decide whether $s \in \mathbb{S}_{O}$ from $s \in \mathbb{S}$ and $O \in \Omega$,

- compute $\tilde{f}\left(s_{1}, \ldots, s_{n}\right)$ from $s_{i} \in \mathbb{S}_{O_{i}}$ and $f \in$ $\mathbb{F}_{O_{0}, O_{1}, \ldots, O_{n}}$,

- decide whether $c \in \mathbb{C}_{O}$ from $c \in \mathbb{C}$ and $O \in \Omega$,

- compute $c \odot s$ from $c \in \mathbb{C}_{O}$ and $s \in \mathbb{S}_{O}$ for any $O \in \Omega$.

- decide whether $s \in \mathcal{L}(G)$ from $s \in \mathbb{S}_{*}$ and $G \in$ $\mathcal{G}(\Sigma)$

Assumption 2. There is $p \in \mathbb{N}$ such that the arity of every $f \in \mathbb{F}$ is at most $p$.

Assumption 3. There are polynomial-time algorithms that compute $\operatorname{SUB}(d), \operatorname{CON}(d)$ and $\operatorname{FUN}(d)$ from $d \in \mathbb{S}_{*}$ such that $\mathbb{S}_{\mid d} \subseteq \operatorname{SUB}(d) \subseteq \mathbb{S}, \mathbb{C}_{\mid d} \subseteq$ $\operatorname{CON}(d) \subseteq \mathbb{C}$ and $\mathbb{F}_{\mid d} \subseteq \operatorname{FUN}(d) \subseteq \mathbb{F}$.

Actually by Assumptions 2 and 3, one can derive the polynomial-time uniform membership decidability. Moreover, it is easy to filter out nonmembers of $\mathbb{S}_{\mid d}, \mathbb{C}_{\mid d}$ and $\mathbb{F}_{\mid d}$ from $\operatorname{SuB}(d), \operatorname{CON}(d)$ and
$\operatorname{Fun}(d)$, respectively, but it is not necessary. Assumption 3 implies $\left|\Omega_{\mid d}\right|$ is polynomially bounded, since $O \in \Omega_{\mid d}$ iff $\mathbb{F}_{O, O_{1}, \ldots, O_{n}} \neq \varnothing$ for some $O_{1}, \ldots, O_{n}$.

We write $\operatorname{Sub}_{O}(D)=\operatorname{Sub}(D) \cap \mathbb{S}_{O}$, $\operatorname{CoN}_{O}(D)=\operatorname{CoN}(D) \cap \mathbb{C}_{O}$ and $\operatorname{FuN}_{\vec{O}}(D)=$ $\operatorname{FuN}(D) \cap \mathbb{F}_{\vec{O}}$.

It is often the case that elements of $\Omega$ represents pairwise disjoint sets. Actually for any signature $\Sigma$, one can find $\Sigma^{\prime}=\left\langle\Omega^{\prime}, \mathbb{F}^{\prime}, O_{*}\right\rangle$ that satisfies this condition such that $\mathcal{L}(\Sigma)=\mathcal{L}\left(\Sigma^{\prime}\right)$. Let $\Omega^{\prime}=\left\{O^{\prime} \mid O \in \Omega\right\} \cup\left\{O_{*}\right\}$ and $\mathbb{O}_{O^{\prime}}=\mathbb{O}_{O} \times\{O\}$ for each $O \in \Omega-\left\{O_{*}\right\}$. For $f \in \mathbb{F}_{O_{0}, \ldots, O_{n}}$ with $\tilde{f}\left(s_{1}, \ldots, s_{n}\right)=s_{0}$, we have $f^{\prime} \in \mathbb{F}_{O_{0}^{\prime}, \ldots, O_{n}^{\prime}}^{\prime}$ with $\tilde{f}^{\prime}\left(s_{1}^{\prime}, \ldots, s_{n}^{\prime}\right)=s_{0}^{\prime}$ where $s_{i}^{\prime}=\left(s_{i}, O_{i}\right)$ if $O_{i} \in$ $\Omega-\left\{O_{*}\right\}$ and $s_{i}^{\prime}=s_{i}$ if $O_{i}=O_{*}$. Clearly every $\Sigma$-grammar has an equivalent $\Sigma^{\prime}$-grammar. Moreover, this makes it clear that from $s \in \mathbb{S}$ one can immediately specify the unique sort $O^{\prime} \in \Omega^{\prime}$ such that $s \in \mathbb{O}_{O^{\prime}}$. Similarly we may assume that each $c \in \mathbb{C}$ has unique $O \in \Omega$ such that $c \in \mathbb{C}_{O}$ and finding that $O$ is a trivial task. Hereafter we work under this assumption. By $O$ and $f$ we mean $\mathbb{O}_{O}$ and $\tilde{f}$ for notational convenience.

Example 1. A right regular grammar over an alphabet $\Delta$ is a $\Sigma_{\text {reg }}$-grammar for $\Sigma_{\text {reg }}=\left\langle\left\{\Delta^{*}\right\}, \mathbb{F}, \Delta^{*}\right\rangle$. $\mathbb{F}$ has nullary functions which are members of $\Delta \cup$ $\{\varepsilon\}$ and unary functions $f_{a}$ for $f_{a}(w)=a w$ for all $w \in \Sigma^{*}$ for some $a \in \Delta$. Clearly the class of right regular grammars satisfies Assumptions 1, 2 and 3.

Example 2. A CFG is a $\Sigma_{\text {cfg }}$-grammar for $\Sigma_{\text {cfg }}=$ $\left\langle\left\{\Delta^{*}\right\}, \mathbb{F}, \Delta^{*}\right\rangle$ where each $f \in \mathbb{F}$ is represented as an $(n+1)$-dimension vector of strings $\left\langle u_{0}, \ldots, u_{n}\right\rangle$ such that $f\left(v_{1}, \ldots, v_{n}\right)=u_{0} v_{1} u_{1} \ldots v_{n} u_{n}$ for all $v_{i} \in \Delta^{*}$. The class of CFGs itself satisfies Assumption 1 but not Assumptions 2 and 3, since we have no limit on $n$. But several normal forms fulfill Assumptions 2 and 3.

Example 3. Let $\Omega=\left\{O_{1}, O_{2}, \ldots\right\}$ where $O_{m}$ denotes the set of $m$-tuples of strings. Linear context-free rewriting systems, equivalent to nondeleting multiple CFGs, are $\Sigma_{\mathrm{mcfg}}$-grammars where $O_{*}=O_{1}=\Delta^{*}$ and every $f \in \mathbb{F}_{O_{m_{0}}, O_{m_{1}}, \ldots, O_{m_{n}}}$ concatenates strings $u_{i, j}$ occurring in an input $\left\langle\left\langle u_{1,1}, \ldots, u_{1, m_{1}}\right\rangle, \ldots,\left\langle u_{n, 1}, \ldots, u_{n, m_{n}}\right\rangle\right\rangle$ in some way to form an $m_{0}$-tuple of strings. The uniform membership problem of this class is PSPACE- 
complete (Kaji et al., 1992). There are infinitely many ways to decompose a string $d$ into substructures and contexts as $O_{m} \in \Omega_{\mid d}$ for all $m$. Assumptions 1 and 3 will be fulfilled when we restrict admissible functions so that $\mathbb{F}_{O_{m_{0}}, \ldots, O_{m_{n}}} \neq \varnothing$ only if $n \leq p$ and $m_{i} \leq q$ for all $i$.

As is the case for multiple CFGs, Assumption 2 is often needed to make the uniform membership problem solvable in polynomial-time (Assumption 1).

\section{Learning models}

Learning algorithms in this paper work under three different learning models.

A positive presentation (text) of a language $L_{*} \subseteq$ $\mathbb{O}_{*}$ is an infinite sequence $d_{1}, d_{2}, \cdots \in \mathbb{O}_{*}$ such that $L_{*}=\left\{d_{i} \mid i \geq 1\right\}$. In the framework of identification in the limit from positive data, a learner is given a positive presentation of the language $L_{*}=\mathcal{L}\left(G_{*}\right)$ of the target grammar $G_{*}$ and each time a new example $d_{i}$ is given, it outputs a grammar $G_{i}$ computed from $d_{1}, \ldots, d_{i}$. We say that a learning algorithm $\mathcal{A}$ identifies $G_{*}$ in the limit from positive data if for any positive presentation $d_{1}, d_{2}, \ldots$ of $\mathcal{L}\left(G_{*}\right)$, there is an integer $n$ such that $G_{n}=G_{m}$ for all $m \geq n$ and $\mathcal{L}\left(G_{n}\right)=\mathcal{L}\left(G_{*}\right)$. We say that $\mathcal{A}$ identifies a class $\mathbb{G}$ of grammars in the limit from positive data iff $\mathcal{A}$ identifies all $G \in \mathbb{G}$ in the limit from positive data.

We say that $\mathcal{A}$ identifies a class $\mathbb{G}$ of grammars in the limit from positive data and membership queries when we allow $\mathcal{A}$ to ask membership queries (MQs) to an oracle when it computes a hypothesis grammar. An instance of an MQ is an object $d \in \mathbb{O}_{*}$ and the oracle answers whether $d \in L_{*}$ in constant time.

The third model is the learning with a minimally adequate teacher (MAT). A learner is not given a positive presentation but it may ask equivalence queries (EQs) to an oracle in addition to MQs. An instance of an EQ is a grammar $G$. If $\mathcal{L}(G)=L_{*}$, the oracle answers "Congratulations!" and the learning process ends. Otherwise, the oracle returns a counerexample $d \in\left(L_{*}-\mathcal{L}(G)\right) \cup\left(\mathcal{L}(G)-L_{*}\right)$, which is called positive if $d \in L_{*}-\mathcal{L}(G)$ and negative if $d \in \mathcal{L}(G)-L_{*}$.

When we have an oracle, the learning task itself is trivial unless we show some favorable property on the learning efficiency.

\section{Learnable subclasses}

This section presents how $\Sigma$-grammars with distributional properties can be learned. Note that all of those properties are relative to $\Sigma$. We assume $\Sigma$ grammars $G_{*}=\left\langle N_{*}, \sigma_{*}, F_{*}, P_{*}, I_{*}\right\rangle$ in this section have no useless nonterminals or functions. That is, $\mathcal{S}\left(G_{*}, X\right) \neq \varnothing, \mathcal{C}\left(G_{*}, X\right) \neq \varnothing$ for all $X \in N_{*}$ and every $f \in F_{*}$ appears in some rule in $P_{*}$.

\subsection{Substitutable Languages}

Definition 1 (Clark and Eyraud (2007)). A language $L \in \mathcal{L}(\Sigma)$ is said to be substitutable if for any $O \in$ $\Omega, c_{1}, c_{2} \in \mathbb{C}_{O}$ and $s_{1}, s_{2} \in \mathbb{S}_{O}$,

$c_{1} \odot s_{1}, c_{1} \odot s_{2}, c_{2} \odot s_{1} \in L$ implies $c_{2} \odot s_{2} \in L$.

The definition can be rephrased as follows:

$$
s_{1}^{\ddagger} \cap s_{2}^{\ddagger} \neq \varnothing \text { implies } s_{1} \equiv_{L} s_{2} .
$$

Example 4. Yoshinaka (2008) has proposed a learning algorithm for $k, l$-substitutable CFLs, which satisfy the following property:

$$
\begin{aligned}
x_{1} u y_{1} v z_{1}, x_{1} u y_{2} v z_{1}, x_{2} u y_{1} v z_{2} & \in L \\
\Longrightarrow & x_{2} u y_{2} v z_{2} \in L
\end{aligned}
$$

for any $x_{i}, y_{i}, z_{i} \in \Delta^{*}, u \in \Delta^{k}$ and $v \in \Delta^{l}$. We define a signature $\Sigma_{k, l}=\left\langle\Omega_{k, l}, \mathbb{F}_{k, l}, O_{*}\right\rangle$ as follows. Let $\Omega_{k, l}=\left\{O_{*}\right\} \cup\left\{O_{u, v} \mid u \in \Delta^{k}\right.$ and $v \in$ $\left.\Delta^{l}\right\} \cup\left\{O_{u} \mid u \in \Delta^{<k+l}\right\}$, where $O_{*}=\Delta^{*}, O_{u, v}=$ $\left\{\overline{u w v} \mid w \in \Delta^{*}\right\}$ and $O_{u}=\{\bar{u}\}$. Here we put overlines to make elements of $\Omega$ pairwise disjoint. Let $\mathbb{F}_{k, l}=\left\{+_{\alpha, \beta} \mid \alpha, \beta \in \Omega-\left\{\Delta^{*}\right\}\right\} \cup\left\{\square_{\alpha}^{O_{*}} \mid\right.$ $\left.\alpha \in \Omega-\left\{O_{*}\right\}\right\} \cup \Delta$. The binary function $+_{\alpha, \beta}$ concatenates two strings from sorts $\alpha$ and $\beta$ and gives the right sort in $\Omega-\left\{O_{*}\right\}$. For example, $+_{\alpha, \beta} \in \mathbb{F}$ with $\alpha=O_{u, v}, \beta=O_{w}$ has codomain $O_{u, x}$ where $x$ is the suffix of $v w$ of length $l$. The unary operation $\square_{\alpha}^{O_{*}} \in \mathbb{F}_{O_{*}, \alpha}$ simply removes the overline and "promotes" $\bar{u} \in \alpha$ to $u \in O_{*} . \Delta$ consists of the nullary functions giving a single letter from $\bar{\Delta}$. It is not hard to see that every CFG has an equivalent $\Sigma_{k, l^{-}}$ grammar. Note that $O_{*}$-nonterminals never occur on the right hand side of a rule in a $\Sigma_{k, l}$-grammar. Hence $\mathbb{C}_{O_{*}}$ is just the singleton $\left\{\square_{O_{*}}\right\}$ such that $\square_{O_{*}} \odot u=u$ for all $u \in \Delta^{*}$, whereas $\mathbb{C}_{\alpha} \neq \mathbb{C}_{O_{*}}$ contains arbitrary pairs of strings $\langle l, r\rangle \in \Delta^{*} \times \Delta^{*}$ such that $\langle l, r\rangle \odot \bar{u}=l u r$ for any $\bar{u} \in \alpha$. The $\Sigma_{k, l^{-}}$ substitutability is exactly the $k, l$-substitutability. 
Theorem 1. The class of substitutable $\Sigma$-languages is identifiable in the limit from positive data.

The theorem follows Lemmas 2 and 3 below.

From a finite set $D$ of positive examples, Algorithm 1 computes the grammar $\operatorname{SuBSTP}(D)=$ $\langle N, \sigma, F, P, I\rangle$ defined as follows:

- $N_{O}=\left\{\llbracket s \rrbracket \mid s \in \operatorname{SuB}_{O}(D)\right\}$ for $O \in \Omega_{\mid D}$,

- $I=\{\llbracket s \rrbracket \mid s \in D\}$,

- $F=\operatorname{Fun}(D)$,

- $P$ consists of the rules of the form

$$
\llbracket s_{0} \rrbracket \leftarrow f\left\langle\llbracket s_{1} \rrbracket, \ldots, \llbracket s_{n} \rrbracket\right\rangle
$$

where $f \in \operatorname{FUN}_{O_{0}, \ldots, O_{n}}(D)$ for $\llbracket s_{i} \rrbracket \in N_{O_{i}}$ if there is $c \in \operatorname{CoN}_{O}(D)$ such that

$$
c \odot s_{0}, c \odot f\left(s_{1}, \ldots, s_{n}\right) \in D .
$$

Since we assume elements of $\Omega$ are pairwise disjoint, each $\llbracket s \rrbracket$ belongs to a unique sort. Otherwise, each nonterminal should be tagged with a sort like $\llbracket s, O \rrbracket$.

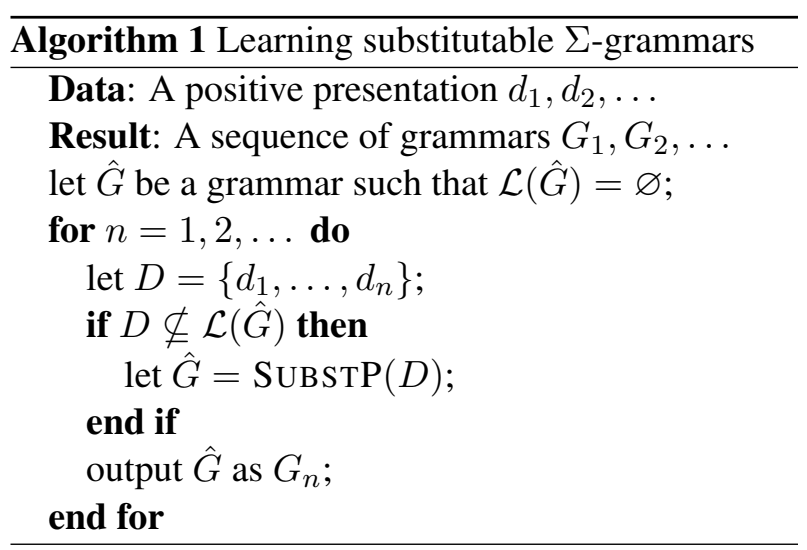

An alternative way to construct a grammar is to use contexts rather than substructures for nonterminals. One can replace $\operatorname{SuBSTP}(D)$ in the algorithm by $\operatorname{SubstD}(D)$ which is defined as follows.

- $N_{O}=\left\{\llbracket c \rrbracket \mid c \in \operatorname{CoN}_{O}(D)\right\}$ for $O \in \Omega_{\mid D}$,

- $I=\left\{\llbracket \square \square_{O_{*}} \rrbracket\right\}$,

- $F=\mathrm{FuN}(D)$,

- $P$ consists of the rules of the form

$$
\llbracket c_{0} \rrbracket \leftarrow f\left\langle\llbracket c_{1} \rrbracket, \ldots, \llbracket c_{n} \rrbracket\right\rangle
$$

where $f \in \mathrm{FUN}_{O_{0}, \ldots, O_{n}}$ for $\llbracket c_{i} \rrbracket \in N_{O_{i}}$ if there are $s_{i} \in \operatorname{SuB}_{O_{i}}(D)$ such that

$c_{i} \odot s_{i} \in D$ for all $i$ and $c_{0} \odot f\left(s_{1}, \ldots, s_{n}\right) \in D$.
The existing algorithms for different classes of substitutable languages (Clark and Eyraud, 2007; Yoshinaka, 2008; Yoshinaka, 2011a) are based on slight variants of SUBSTP. This paper shows the correctness of the algorithm using SUBSTD.

Lemma 2. Let $D$ be a finite subset of a $\Sigma$ substitutable language $L_{*}$ and $G$ the grammar output by $\operatorname{SuBSTD}(D)$. Then $\mathcal{L}(G) \subseteq L_{*}$.

Proof. One can show by induction on the derivation that if $s \in \mathcal{S}(G, \llbracket c \rrbracket)$ then $c \odot s \in L_{*}$. Suppose that $G$ has a rule $\llbracket c \rrbracket \leftarrow f\left\langle\llbracket c_{1} \rrbracket, \ldots, \llbracket c_{n} \rrbracket\right\rangle$, $s_{i} \in \mathcal{S}\left(G, \llbracket c_{i} \rrbracket\right)$ and $s=f\left(s_{1}, \ldots, s_{n}\right)$. The induction hypothesis says $c_{i} \odot s_{i} \in L_{*}$ for all $i$. By the rule construction, there are $t_{i}$ for $i=1, \ldots, n$ such that $c_{i} \odot t_{i} \in D \subseteq L_{*}$ and $c \odot f\left(t_{1}, \ldots, t_{n}\right) \in D \subseteq L_{*}$. We have $s_{i} \equiv_{L_{*}} t_{i}$ since they occur in the same context $c_{i}$. By Lemma $1, c \odot f\left(s_{1}, \ldots, s_{n}\right) \in L_{*}$.

Let $G_{*}=\left\langle N_{*}, \sigma_{*}, F_{*}, P_{*}, I_{*}\right\rangle$ be a $\Sigma$-grammar generating $L_{*}$. Fix $s_{X} \in \mathcal{S}\left(G_{*}, X\right)$ and $c_{X} \in$ $\mathcal{C}\left(G_{*}, X\right)$ where $c_{X}=\square_{O_{*}}$ for $X \in I_{*}$. Define $D_{*}$ by

$$
\begin{aligned}
D_{*}= & \left\{c_{X} \odot s_{X} \mid X \in N_{*}\right\} \\
\cup\left\{c_{X_{0}} \odot f\left(s_{X_{1}}, \ldots, s_{X_{n}}\right)\right. & \left.\mid X_{0} \leftarrow f\left\langle X_{1}, \ldots, X_{n}\right\rangle \in P_{*}\right\} .
\end{aligned}
$$

Lemma 3. If $D_{*} \subseteq D$, then $\mathcal{S}\left(G_{*}, X\right) \subseteq$ $\mathcal{S}\left(\operatorname{SUBSTD}(D), \llbracket s_{X} \rrbracket\right)$ for all $X$.

Proof. Let $G=\operatorname{SubstD}(D)$. If $G_{*}$ has a rule $X_{0} \leftarrow f\left\langle X_{1}, \ldots, X_{n}\right\rangle$ then $G$ has the corresponding rule $\llbracket c_{X_{0}} \rrbracket \leftarrow f\left\langle\llbracket c_{X_{1}} \rrbracket, \ldots, \llbracket c_{X_{n}} \rrbracket\right\rangle$, since

$$
c_{X_{0}} \odot f\left(s_{X_{1}}, \ldots, s_{X_{n}}\right), c_{X_{i}} \odot s_{X_{i}} \in D .
$$

In particular since $c_{X}$ for $X \in I$ is the identity function $\square_{O_{*}}$, the corresponding nonterminal $\llbracket c_{X} \rrbracket=$ $\llbracket \square_{O_{*}} \rrbracket$ is the initial symbol of $G$, too.

This shows that we do not need too many data to achieve a right grammar, since $\left|D_{*}\right| \leq\left|P_{*}\right|+\left|N_{*}\right|$, where $|\cdot|$ denotes the cardinality of a set. Moreover, it is easy to see Algorithm 1 updates its conjecture in polynomial time in the total size of $D$ by Assumptions 1,2 and 3 . 


\subsection{Finite kernel property}

Definition 2 (Clark et al. (2009), Yoshinaka (2011b)). A nonempty finite set $S \subseteq \mathbb{S}_{\sigma(X)}$ is called a $k$-kernel of a nonterminal $X$ if $|S| \leq k$ and

$$
\mathcal{S}(G, X) \equiv_{\mathcal{L}(G)} S
$$

A $\Sigma$-grammar $G$ is said to have the $k$-finite kernel property $(k$-FKP) if every nonterminal $X$ has a $k$ kernel $S_{X}$.

Theorem 2. Under Assumptions 1, 2 and 3, Algorithm 2 identifies $\Sigma$-grammars with the $k$-FKP in the limit from positive data and membership queries.

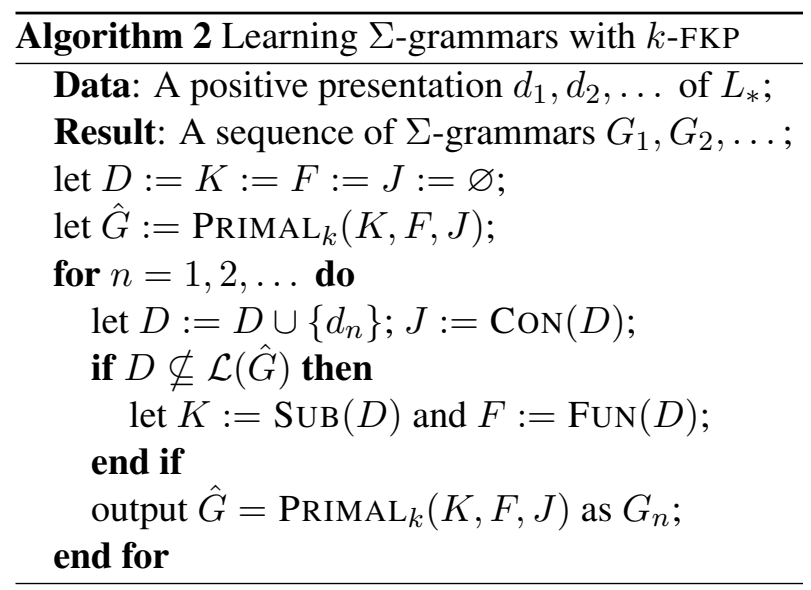

The conjecture grammar $\operatorname{PRIMAL}_{k}(K, F, J)=$ $\langle N, \sigma, F, P, I\rangle$ of Algorithm 2 is defined from finite sets of substructures $K \subseteq \mathbb{S}$, functions $F \subseteq \mathbb{F}$ and contexts $J \subseteq \mathbb{C}$. The subsets of those sets corresponding to respective sorts are denoted as $K_{O}=$ $K \cap \mathbb{S}_{O}, J_{O}=J \cap \mathbb{C}_{O}$ and $F_{\vec{O}}=F \cap \mathbb{F}_{\vec{O}}$.

- $N_{O}=\left\{\llbracket S \rrbracket \mid S \subseteq K_{O}\right.$ with $\left.1 \leq|S| \leq k\right\}$ for each $O \in \Omega_{\mid D}$,

- $I=\left\{\llbracket S \rrbracket \in N_{O_{*}} \mid S \subseteq L\right\}$,

- $P$ consists of the rules of the form

$$
\llbracket S_{0} \rrbracket \leftarrow f\left\langle\llbracket S_{1} \rrbracket, \ldots, \llbracket S_{n} \rrbracket\right\rangle
$$

where $f \in F_{O_{0}, O_{1}, \ldots, O_{n}}$ for $\llbracket S_{i} \rrbracket \in N_{O_{i}}$ if

$$
\left(S_{0}^{\ddagger} \cap J_{O_{0}}\right) \odot f\left(S_{1}, \ldots, S_{n}\right) \subseteq L_{*} .
$$

The grammar is constructed by the aid of finitely many MQs. $\operatorname{PRIMAL}_{k}(K, F, J)$ can be computed in polynomial time by Assumptions 1, 2 and 3 . A rule $\llbracket S_{0} \rrbracket \leftarrow f\left\langle\llbracket S_{1} \rrbracket, \ldots, \llbracket S_{n} \rrbracket\right\rangle$ is compatible with the semantics of the nonterminals if $S_{0}^{\dagger} \supseteq$ $f\left(S_{1}^{\dagger}, \ldots, S_{n}^{\dagger}\right)$, which is equivalent to

$$
S_{0}^{\ddagger} \odot f\left(S_{1}, \ldots, S_{n}\right) \subseteq L_{*}
$$

by Lemma 1. However, this condition (2) cannot be checked by finitely many MQs. The condition (1) can be seen as an approximation of (2), which is decidable by finitely many MQs. Clearly (2) implies (1) but not vice versa. If a rule satisfies (1) but not (2), we call the rule incorrect. If a rule is incorrect, there is a witness $c \in \mathbb{C}_{O_{0}}-J_{O_{0}}$ such that $c \odot S_{0} \in L_{*}$ and $c \odot f\left(S_{1}, \ldots, S_{n}\right) \notin L_{*}$.

Lemma 4. For every finite $K \subseteq \mathbb{S}$ and $F \subseteq \mathbb{F}$ there is $J \subseteq \mathbb{C}$ such that $\hat{G}=\operatorname{PrimaL}(K, F, J)$ has no incorrect rules and $|J| \leq|F||K|^{k(p+1)}$, in which case $\mathcal{L}(\hat{G}) \subseteq L_{*}$.

Let $S_{X}$ be a $k$-kernel of each nonterminal $X$ of a grammar $G_{*}=\left\langle N_{*}, \sigma_{*}, F_{*}, P_{*}, I_{*}\right\rangle$ generating $L_{*}$.

Lemma 5. There is a finite subset $D \subseteq L_{*}$ such that $S_{X} \subseteq \mathbb{S}_{\mid D}$ for all $X \in N_{*}, F_{*} \subseteq \mathbb{F}_{\mid D}$ and $|D| \leq k\left|N_{*}\right|+\left|P_{*}\right|$. Moreover, if $S_{X} \subseteq K$ for all $X \in N_{*}$ and $F_{*} \subseteq F$, then $L_{*} \subseteq \mathcal{L}(\hat{G})$.

We prove Theorem 2 discussing the efficiency.

Proof of Theorem 2. Clearly Algorithm 2 updates its conjecture in polynomial time in the data size. Polynomially (in the size of $G_{*}$ ) many positive examples will stabilize $K$ and $F$ by Lemma 5. After $K$ and $F$ stabilized, all the incorrect rules will be removed with at most polynomially (in $|K||F|$ ) many examples by Lemma 4. After that point Algorithm 2 never changes the conjecture, which generates the target language $L_{*}$.

\subsection{Congruential grammars}

Definition 3 (Clark (2010a)). A $\Sigma$-grammar $G$ is said to be congruential if every $s \in \mathcal{S}(G, X)$ is a 1-kernel of every $X \in N$.

Congruential $\Sigma$-grammars have the 1-FKP. Under the following additional assumption, this special case will be polynomial-time learnable with a minimally adequate teacher.

Assumption 4. For any derivation tree $\tau$, the size of its yield $\tilde{\tau}$ is polynomially bounded by that of $\tau$.

Theorem 3. Under Assumptions 1, 2, 3 and 4, Algorithm 3 learns any language $L_{*}$ generated by a 
congruential $\Sigma$-grammar $G_{*}$ with a minimally adequate teacher in time polynomial in $\left|N_{*}\right|,\left|F_{*}\right|, \ell$ where $\ell$ is the total size of counterexamples given to the learner.

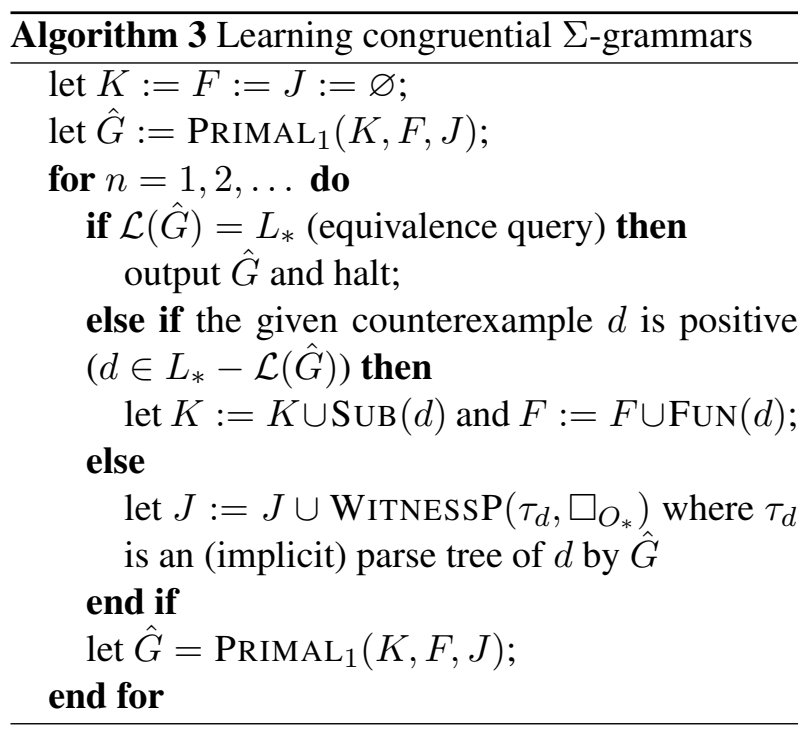

Algorithm 3 uses the same grammar construction PRIMAL as Algorithm 2 where the parameters $K$ and $F$ are calculated from positive counterexamples given by the oracle. On the other hand, $J$ is computed in a different way. By Lemma 4, when the oracle answers a negative counterexample $d$ towards an EQ, our conjecture $\hat{G}$ must use an incorrect rule to derive $d$. To find and remove such an incorrect rule, Algorithm 3 calls a subroutine WITNESSP with input $\left(\tau_{d}, \square\right)$, where $\tau_{d}$ is a derivation tree of $\hat{G}$ whose yield is $d$. To be precise, $\tau_{d}$ does not have to be a derivation tree. Rather what we require is that for each $s \in \mathbb{S}_{\mid d}$, one can compute at least one tuple of $s_{1}, \ldots, s_{n} \in \mathbb{S}_{\mid d}$ and $f \in \mathbb{F}_{\mid d}$ such that $s=f\left(s_{1}, \ldots, s_{n}\right)$ and the height of the lowest derivation tree of each $s_{i}$ is strictly lower than that of $s$. Indeed one can do this in polynomial time by a dynamic programming method from $\operatorname{SuB}(d)$ and $\operatorname{Fun}(d)$. Yet for explanatory easiness, we treat such information as an (implicit) derivation tree $\tau_{d}$. The procedure WITNESSP returns a context that witnesses an incorrect rule that contributes to generating $d$ by searching $\tau_{d}$ recursively calling itself. The procedure WITNESSP in general takes a pair $(\tau, c)$ such that $\tau$ is an $\llbracket s \rrbracket$-derivation tree of $\hat{G}$ and $c \in s^{\ddagger}-\tilde{\tau}^{\ddagger}$. Let $\tau=\rho\left(\tau_{1}, \ldots, \tau_{n}\right)$ where $\rho=$ $\llbracket s \rrbracket \leftarrow f\left\langle\llbracket s_{1} \rrbracket, \ldots, \llbracket s_{n} \rrbracket\right\rangle$. If $c \odot f\left(s_{1}, \ldots, s_{n}\right) \notin L_{*}$ then the rule $\rho$ is incorrect. So WITNESSP returns $c$ which witnesses the incorrectness of the rule. Otherwise, we have

$$
\begin{aligned}
& c \odot f\left(s_{1}, \ldots, s_{n}\right) \in L_{*} \\
& c \odot f\left(\tilde{\tau}_{1}, \ldots, \tilde{\tau}_{n}\right) \notin L_{*}
\end{aligned}
$$

for the yields $\tilde{\tau}_{i}$ of $\tau_{i}$. One can find $i$ such that

$$
\begin{aligned}
& c \odot f\left(s_{1}, \ldots, s_{i-1}, s_{i}, \tilde{\tau}_{i+1}, \ldots, \tilde{\tau}_{n}\right) \in L_{*} \\
& c \odot f\left(s_{1}, \ldots, s_{i-1}, \tilde{\tau}_{i}, \tilde{\tau}_{i+1}, \ldots, \tilde{\tau}_{n}\right) \notin L_{*} .
\end{aligned}
$$

This means an incorrect rule is in $\tau_{i}$. We call $\operatorname{WitnessP}\left(\tau_{i}, c \odot f\left(s_{1}, \ldots, s_{i-1}, \square, \tilde{\tau}_{i+1}, \ldots, \tilde{\tau}_{n}\right)\right)$.

Lemma 6. The procedure $\operatorname{WiTnEssP}\left(\tau_{d}, \square\right)$ runs in polynomial time in $\ell$ and $|d|$.

Proof. The number of recursive calls of WITNESSP is no more than the height of $\tau_{d}$, which is at most $\left|\mathbb{S}_{\mid d}\right|$. Let the instance of the $j$-th recursive call be $\left(\tau_{j}, c_{j}\right)$ and $\chi_{j}$ the derivation context for $c=\tilde{\chi}_{j}$. $\chi_{j+1}$ is obtained from $\chi_{j}$ by replacing at most $p$ subtrees by a derivation tree whose yield is an element of $K$. By Assumption 4, the size of $c_{j}$ and thus the size of an instance of an MQ is polynomially bounded by $|d| \ell$. WITNESSP runs in polynomial time.

Lemma 7. Each time Algorithm 3 receives a negative counterexample, at least one incorrect rule is removed.

Lemma 8. Let $G_{*}=\left\langle N_{*}, \sigma, F_{*}, P_{*}, I_{*}\right\rangle$ be a congruential grammar generating $L_{*}$. Each time Algorithm 3 receives a positive counterexample, the cardinality of the set $\left\{X \in N_{*} \mid K \cap \mathcal{L}\left(G_{*}, X\right)=\varnothing\right\}$ $\cup\left(F_{*}-F\right)$ decreases strictly.

Proof of Theorem 3. Time between an EQ and another is polynomially bounded by Lemma 6 . By Lemmas 5 and 8, Algorithm 3 gets at most $\left|N_{*}\right|+$ $\left|F_{*}\right|$ positive counterexamples. The grammar $\hat{G}=$ $\operatorname{PrImAL}(K, F, J)$ is constructed from those positive counterexamples, so it has polynomially many rules. Therefore, by Lemma 7, after getting polynomially many negative counterexamples, which suppress all the incorrect rules, Algorithm 3 gets a right grammar representing $L_{*}$. 


\subsection{Finite context property}

Definition 4 (Clark (2010b), Yoshinaka $\left.(2011 b)^{1}\right)$. A nonempty finite set $C \subseteq \mathbb{C}$ is called a $k$-context of a nonterminal $X$ if $|C| \leq k$ and

$$
\mathcal{S}(G, X) \equiv_{\mathcal{L}(G)} C^{\dagger} .
$$

A $\Sigma$-grammar $G$ is said to have the $k$-(weak) finite context property ( $k$-FCP) if every nonterminal $X$ has a $k$-context $C_{X}$.

Theorem 4. Under Assumptions 1, 2 and 3, Algorithm 4 identifies $\Sigma$-grammars with the $k$-FCP in the limit from positive data and membership queries.

The theorem can be shown by an argument similar to the proof of Theorem 2 based on Lemmas 9 and 10 below. The discussion on the learning efficiency of Algorithm 2 is applied to Algorithm 4 as well.

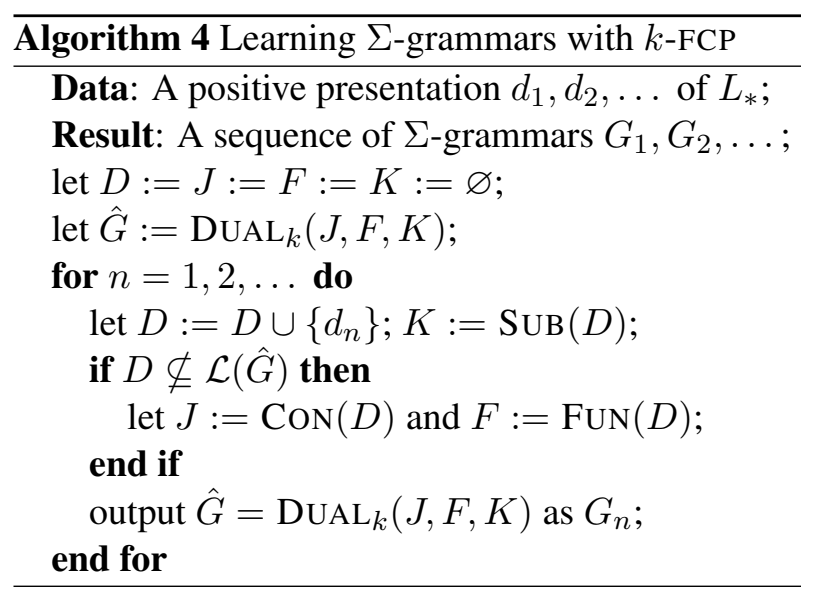

The conjecture grammar $\operatorname{DuAL}_{k}(J, F, K)=$ $\langle N, \sigma, F, P, I\rangle$ of Algorithm 4 is defined from finite sets of contexts $J \subseteq \mathbb{C}$, functions $F \subseteq \mathbb{F}$ and substructures $K \subseteq \mathbb{S}$. For each $C \subseteq J_{O}$, we write $C^{(K)}$ to mean $C^{\dagger} \cap K_{O}$. This set can be seen as a finite approximation of $C^{\dagger}$, which is computable with MQs.

- $N_{O}=\left\{\llbracket C \rrbracket \mid C \subseteq J_{O}\right.$ with $\left.1 \leq|C| \leq k\right\}$ for $O \in \Omega_{\mid D}$,

- $I=\left\{\llbracket\left\{\square_{*}\right\} \rrbracket\right\}$,

- $P$ consists of the rules of the form

$$
\llbracket C_{0} \rrbracket \leftarrow f\left\langle\llbracket C_{1} \rrbracket, \ldots, \llbracket C_{n} \rrbracket\right\rangle
$$

where $f \in F_{O_{0}, \ldots, O_{n}}$ for $\llbracket C_{i} \rrbracket \in N_{O_{i}}$ if $C_{0} \odot$ $f\left(C_{1}^{(K)}, \ldots, C_{m}^{(K)}\right) \subseteq L_{*}$.

\footnotetext{
${ }^{1}$ We adopt the definition by Yoshinaka, which is slightly weaker than Clark's.
}

We say that a rule $\llbracket C_{0} \rrbracket \leftarrow f\left(\llbracket C_{1} \rrbracket, \ldots, \llbracket C_{n} \rrbracket\right)$ is incorrect if $C_{0} \odot f\left(C_{1}^{\dagger}, \ldots, C_{n}^{\dagger}\right) \nsubseteq L_{*}$. In that case, there are $s_{i} \in C_{i}^{\dagger}$ such that $C_{0} \odot f\left(s_{1}, \ldots, s_{n}\right) \nsubseteq L_{*}$.

Lemma 9. For every finite $J \subseteq \mathbb{C}$ and $F \subseteq \mathbb{F}$ there is $K \subseteq \mathbb{S}$ such that $\hat{G}=\operatorname{DUAL}(J, F, K)$ has no incorrect rules and $|K| \leq p|F||J|^{k(p+1)}$, in which case $\mathcal{L}(\hat{G}) \subseteq L_{*}$.

Let $G_{*}=\left\langle N_{*}, \sigma_{*}, F_{*}, P_{*}, I_{*}\right\rangle$ generate $L_{*}$ and $C_{X}$ a $k$-context of each nonterminal $X \in N_{*}$.

Lemma 10. There is a finite subset $D \subseteq L_{*}$ such that $C_{\mid D} \supseteq C_{X}$ for all $X \in N_{*}, F_{\mid D} \supseteq F_{*}$ and $|D| \leq k\left|N_{*}\right|+\left|P_{*}\right|$. Moreover, if $J \supseteq C_{X}$ for all $X \in N_{*}$ and $F \supseteq F_{*}$, then $L_{*} \subseteq \mathcal{L}(\hat{G})$.

\subsection{Context-deterministic grammars}

Definition 5 (Shirakawa and Yokomori (1993), Yoshinaka $\left.(2012)^{2}\right)$. A $\Sigma$-grammar $G$ is said to be (weakly) context-deterministic if every $c \in \mathcal{C}(G, X)$ is a 1-context of every $X \in N_{O}$.

Differently from Theorem 3, we do not need Assumption 4 for learning context-deterministic grammars with a minimally adequate teacher.

Theorem 5. Under Assumptions 1, 2 and 3, Algorithm 3 learns any language $L_{*}$ generated by a context-deterministic $\Sigma$-grammar $G_{*}$ with a minimally adequate teacher in time polynomial in $\left|N_{*}\right|,\left|F_{*}\right|, \ell$ where $\ell$ is the total size of counterexamples given to the learner.

Proof. By Lemmas 11, 12 and 13 below.

Algorithm 5 uses the same grammar construction DuAL as Algorithm 4. By Lemma 9, when the oracle answers a negative counterexample $d$ towards an EQ, our conjecture $\hat{G}$ must use an incorrect rule to derive $d$. To find and remove such an incorrect rule, Algorithm 5 calls a subroutine WiTNESSD with a derivation tree $\tau_{d}$ of $\hat{G}$ whose yield is $d$. The procedure WITNESSD returns a finite set of substructures that witnesses an incorrect rule that contributes to generating $d$. An input given to WITNESSD is in general a $\llbracket c \rrbracket$-derivation tree $\tau$ such that $c \odot \tilde{\tau} \notin L_{*}$. Let $\tau=\rho\left[\tau_{1}, \ldots, \tau_{n}\right]$ where $\rho=\llbracket c \rrbracket \leftarrow f\left\langle\llbracket c_{1} \rrbracket, \ldots, \llbracket c_{n} \rrbracket\right\rangle$. If there is $i$ such that $c_{i} \odot \tilde{\tau}_{i} \notin L_{*}$, we recursively call $\operatorname{WiTNESSD}\left(\tau_{i}\right)$.

\footnotetext{
${ }^{2}$ We adopt the definition by Yoshinaka, which is slightly weaker than Shirakawa and Yokomori's.
} 


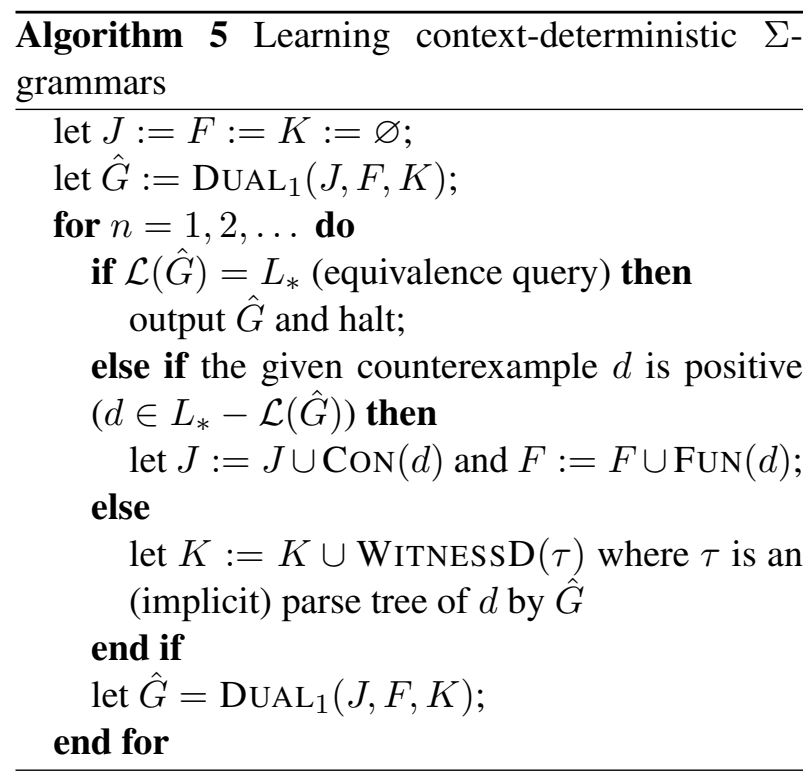

Otherwise, $\tilde{\tau}_{i} \in c_{i}^{\dagger}$ for all $i$, which means the rule $\rho$ is incorrect. WITNESSD $(\tau)$ returns the set $\left\{\tilde{\tau}_{1}, \ldots, \tilde{\tau}_{n}\right\}$. Differently from the case of WITNESSP, an instance of a recursive call is always an (implicit) derivation tree of some $s \in \mathbb{S}_{\mid d}$. This explains why we do not need Assumption 4 in this case.

Lemma 11. Time between an $\mathrm{EQ}$ and another is polynomially bounded.

Lemma 12. Each time Algorithm 5 receives a negative counterexample, at least one incorrect rule is removed.

Lemma 13. Let $G_{*}=\left\langle N_{*}, \sigma, F_{*}, P_{*}, I_{*}\right\rangle$ be a context-deterministic grammar for $L_{*}$. Each time Algorithm 5 receives a positive counterexample, the set $\left\{X \in N_{*} \mid J \cap \mathcal{C}\left(G_{*}, X\right)=\varnothing\right\} \cup\left(F_{*}-F\right)$ gets shrunk.

\subsection{Combined approaches}

By combining primal and dual approaches, one can obtain stronger approaches (Yoshinaka, 2012). The class of $\Sigma$-grammars whose nonterminals admit either a $k$-kernel or $l$-context can be learned by combining the techniques presented in Sections 6.2 and 6.4 under Assumptions 1, 2 and 3. Also $\Sigma$-grammars whose nonterminals satisfy either the requirement to be congruential or to be contextdeterministic can be learned with a minimally adequate teacher under Assumptions 1, 2, 3 and 4 (Sections 6.3 and 6.5).

\section{Restricted cases}

In some grammar classes, it may be the case that only (supersets of) $\mathbb{C}_{\mid d}$ and $\mathbb{F}_{\mid d}$ are computable in polynomial-time but $\mathbb{S}_{\mid d}$ is not, or the other way around: $\mathbb{S}_{\mid d}$ and $\mathbb{F}_{\mid d}$ are efficiently computable but $\mathbb{C}_{\mid d}$ is not. For example, in non-permuting parallel multiple CFGs (Seki et al., 1991), elements of $\mathbb{S}_{\mid d}$ for a string $d$ are tuples of strings of the form $\left\langle v_{1}, \ldots, v_{m}\right\rangle$ for $d=u_{0} v_{1} u_{1} \ldots v_{m} u_{m}$ and such substrings are polynomially many if $m$ is fixed. However, $\mathbb{C}_{\mid d}$ contains exponentially many contexts. Clark and Yoshinaka (2014) showed that still a dual approach works for parallel multiple CFGs if nonterminals are known to have $k$-contexts belonging to a certain subset $\mathbf{C} \subseteq \mathbb{C}$ such that $\mathbf{C}_{\mid d}=\mathbb{C}_{\mid d} \cap \mathbf{C}$ is polynomial-time computable. A symmetric result of a primal approach has also been obtained by Kanazawa and Yoshinaka (2015) targeting a certain kind of tree grammars. This section does not postulate Assumption 3.

Definition 6. A $\Sigma$-grammar $G$ is said to have the $(k, \mathbf{S})$-FKP if every nonterminal admits a $k$-kernel which is a subset of $\mathbf{S}$.

Assumption 5. There are polynomial-time algorithms that compute $\operatorname{SUB}(d), \operatorname{CON}(d)$ and $\operatorname{FUN}(d)$ such that $\mathbf{S}_{\mid d} \subseteq \operatorname{SuB}(d) \subseteq \mathbb{S}, \mathbb{C}_{\mid d} \subseteq \operatorname{CoN}(d) \subseteq \mathbb{C}$ and $\mathbb{F}_{\mid d} \subseteq \operatorname{FUN}(d) \subseteq \mathbb{F}$, where $\mathbf{S}_{\mid d}=\mathbf{S} \cap \mathbb{S}_{\mid d}$.

It is not hard to see that Algorithm 2 works for learning $\Sigma$-grammars with $(k, \mathbf{S})$-FKP under Assumptions 1, 2 and 5. All discussions in Section 6.2 hold for this restricted case.

The symmetric definition and assumption are as follows.

Definition 7. A $\Sigma$-grammar $G$ is said to have the $(k, \mathbf{C})$-FCP if every nonterminal admits a $k$-context which is a subset of $\mathbf{C}$.

Assumption 6. There are polynomial-time algorithms that compute $\operatorname{SUB}(d), \operatorname{CON}(d)$ and $\operatorname{FUN}(d)$ such that $\mathbb{S}_{\mid d} \subseteq \operatorname{SuB}(d) \subseteq \mathbb{S}, \mathbf{C}_{\mid d} \subseteq \operatorname{CON}(d) \subseteq \mathbb{C}$ and $\mathbb{F}_{\mid d} \subseteq \operatorname{FUN}(d) \subseteq \mathbb{F}$.

It is not hard to see that under Assumptions 1, 2 and 6, Algorithms 4 work for learning $\Sigma$-grammars with $(k, \mathbf{C})$-FCP $\sum$-grammars. All discussions in Section 6.4 hold for this restricted case.

When learning substitutable languages, even a weaker assumption suffices. 
Assumption 7. There are sets $\mathbf{S} \subseteq \mathbb{S}$ and $\mathbf{C} \subseteq \mathbb{C}$ such that for every nonterminal $X$ of $G \in \mathcal{G}(\Sigma)$, we have $\mathcal{S}(G, X) \cap \mathbf{S} \neq \varnothing$ and $\mathcal{C}(G, X) \cap \mathbf{C} \neq \varnothing$. Moreover, there are polynomial-time algorithms that compute $\operatorname{SuB}(d), \operatorname{CON}(d)$ and $\operatorname{Fun}(d)$ such that $\mathbf{S}_{\mid d} \subseteq \operatorname{SuB}(d) \subseteq \mathbb{S}, \mathbf{C}_{\mid d} \subseteq \operatorname{CON}(d) \subseteq \mathbb{C}$ and $\mathbb{F}_{\mid d} \subseteq \mathrm{FUN}(d) \subseteq \mathbb{F}$.

Under Assumptions 1, 2 and 7, Algorithm 1 works using either SUBSTP or SUBSTD.

On the other hand, the results on the polynomialtime MAT learnability of congruential and contextdeterministic $\Sigma$-grammars do not hold anymore under any of Assumptions 5, 6 and 7.

\section{Extending learnable classes}

This section compares learnable classes of $\Sigma$ languages for different $\Sigma$ with the same special sort $O_{*}$. For $\Sigma_{1}$ and $\Sigma_{2}$ with $\Sigma_{i}=\left\langle\Omega^{i}, \mathbb{F}^{i}, O_{*}\right\rangle$, if $\Omega^{1} \subseteq \Omega^{2}$ and $\mathbb{F}^{1} \subseteq \mathbb{F}^{2}$, every $\Sigma_{1}$-grammar is a $\Sigma_{2}$-grammar, so $\mathcal{L}\left(\Sigma_{1}\right) \subseteq \mathcal{L}\left(\Sigma_{2}\right)$. However, since the distributional properties defined so far are relative to a signature, a $\Sigma_{1}$-grammar with a distributional property under $\Sigma_{1}$ does not necessarily have the corresponding property under $\Sigma_{2}$. Yet if $\mathbb{S}_{O}$ and $\mathbb{C}_{O}$ are preserved by moving from $\Sigma_{1}$ to $\Sigma_{2}$, the distributional properties other than the substitutability are preserved.

Let us define the direct union $\Sigma_{0}=\left\langle\Omega^{0}, \mathbb{F}^{0}, O_{*}\right\rangle$ of arbitrary signatures $\Sigma_{1}$ and $\Sigma_{2}$ by $\Omega^{0}=\left\{O_{*}\right\} \cup$ $\left\{(O, i) \mid O \in \Omega^{i}\right.$ with $\left.i \in\{1,2\}\right\}$ where $\mathbb{O}_{(O, i)}=$ $\{(s, i) \mid s \in O\}$ and $\mathbb{F}^{0}=\left\{\mathbb{G}^{1} \cup \mathbb{G}^{2} \cup\left\{\square_{1}, \square_{2}\right\}\right.$, where $\mathbb{G}^{i}$ is a trivial variant of $\mathbb{F}^{i}$ working on the new domain and codomain of the form $(O, i)$ and $\square_{i}(s, i)=s$ for all $s \in O_{*}$. Then every $\Sigma_{i}$-grammar $G$ can be seen as a special type of $\Sigma_{0}$-grammar by adding a new initial symbol $Z$ and rules of the form $Z \leftarrow \square_{\Sigma_{i}}\langle X\rangle$ for all initial symbols $X$ of $G$. We have $\mathcal{L}\left(\Sigma_{1}\right) \cup \mathcal{L}\left(\Sigma_{2}\right) \subseteq \mathcal{L}\left(\Sigma_{0}\right)$. Every $\Sigma_{i}$-grammar that is congruential, context-deterministic, with the $k$-FKP or with the $k$-FCP for $i=1,2$ can be seen as a $\Sigma_{0}$-grammar with those properties. Note that $\mathbb{C}_{O_{*}}$ is the singleton of the identity function in $\Sigma_{0}$, which means any element of $\mathcal{L}(G)$ is a 1-kernel of the new initial symbol $Z$. In this way, from two signatures, one can obtain a richer learnable class of languages.

The above argument on signature generalization does not hold for substitutable case. Rather the op- posite holds. If $\Omega^{1} \subseteq \Omega^{2}$ and $\mathbb{F}^{1} \subseteq \mathbb{F}^{2}$, then a language substitutable under $\Sigma_{2}$ is substitutable under $\Sigma_{1}$ but not vice versa.

Let us say that $\Sigma_{2}$ is finer than $\Sigma_{1}$ if every sort of $\Omega^{1}$ is partitioned into finite number of sorts in $\Omega^{2}$ and every function of $\mathbb{F}^{2}$ is a subfunction of some function in $\mathbb{F}^{1}$ which accords with the partition. That is, every sort $O$ of $\Omega^{1}$ has a finite set $\Omega_{O}^{2} \subseteq \Omega^{2}$ such that $O=\bigcup \Omega_{O}^{2}$ and $\mathbb{F}_{O_{0}, \ldots, O_{n}}^{1}=\bigcup\left\{\mathbb{F}_{O_{0}^{\prime}, \ldots, O_{n}^{\prime}}^{2}\right.$ $\left.O_{i}^{\prime} \in \Omega_{O_{i}}^{2}\right\}$. For instance, $\Sigma_{k, l}$ is finer than $\Sigma_{k^{\prime}, l^{\prime}}$ for $k^{\prime} \leq k$ and $l^{\prime} \leq l$ in Example 4. If $\Sigma_{2}$ is finer than $\Sigma_{1}, \mathcal{L}\left(\Sigma_{1}\right)=\mathcal{L}\left(\Sigma_{2}\right)$ holds. Every language substitutable under $\Sigma_{1}$ is substitutable under $\Sigma_{2}$ but not vice versa. Moreover, every congruential (resp. context-deterministic) $\Sigma_{1}$-grammar has an equivalent congruential (resp. context-deterministic) $\Sigma_{2}$-grammar but not vice versa.

\section{Grammars with partial functions}

Yoshinaka (2015) showed that a dual approach can be applied to the learning of conjunctive grammars. Conjunctive grammars (Okhotin, 2001) are CFGs extended with the conjunctive operation \& so that one can extract the intersection of the languages of nonterminals. For example, a conjunctive rule $A_{0} \rightarrow$ $A_{1} \& A_{2}$ means that if both $A_{1}$ and $A_{2}$ generate the same string $u$ then so does $A_{0}$. Conjunctive grammars cannot be seen as $\Sigma$-grammars, since the conjunctive operation $\&$ is a partial function whose domain is not represented as the direct product of two sorts, which is not legitimate in the general framework of $\Sigma$-grammars.

A partial signature is a triple $\Pi=\left\langle\Omega, \mathbb{F}, O_{*}\right\rangle$ which is defined in the way similar to a (total) signature but $\mathbb{F}$ may have partial functions. Accordingly contexts in $\mathbb{C}$ will be partial functions. We do not have $\mathcal{C}(G, X) \odot \mathcal{S}(G, X) \subseteq \mathcal{L}(G)$ any more, since $c \odot s$ may not be defined for some elements $c \in \mathcal{C}(G, X)$ and $s \in \mathcal{S}(G, X)$. The correspondence between $O$-concept lattices and $\Sigma$-grammars collapses. This prevents the application of the theory of distributional learning developed in this paper to $\Pi$-grammars. Still we can generalize the discussion on the learning of conjunctive grammars.

Definition 8. A $\Pi$-grammar $G$ is said to have the strong $k$-FCP if for any $X \in N_{O}$, there is a finite set 
$C_{X} \subseteq \mathbb{C}_{O}$ with $\left|C_{X}\right| \leq k$ such that

$$
\mathcal{S}(G, X)=\left\{s \mid c \odot s \in L \text { for all } c \in C_{X}\right\} .
$$

Definition 8 requires every $c \in C_{X}$ to be total on $\mathcal{S}(G, X)$. One can learn П-grammars with the strong $k$-FCP under Assumptions 1, 2 and 6, where $\mathbf{C}$ consists of total functions only. The grammar construction $\mathrm{DUAL}_{k}$ should be modified so that we have a rule $\llbracket C_{0} \rrbracket \leftarrow f\left\langle\llbracket C_{1} \rrbracket, \ldots, \llbracket C_{n} \rrbracket\right\rangle$ if $c \odot$ $f\left(s_{1}, \ldots, s_{n}\right) \in L_{*}$ for any $c \in C_{0}$ and $s_{i} \in C_{i}^{(K)}$ such that $f\left(s_{1}, \ldots, s_{n}\right)$ is defined. One might think that one can naturally define context-deterministic grammars accordingly: Every $c \in \mathcal{C}(G, X)$ should be a 1-context of $X$. However, this means that functions in such a $\Pi$-grammar are essentially total.

\section{Acknowledgments}

The view presented in this paper has been sharpened through the interactions and discussions with several researchers with whom I worked on the distributional learning of generalized CFGs. I would like to show my deepest gratitude to Alexander Clark, Makoto Kanazawa, Anna Kasprzik and Gregory Kobele. Without those people this work would have been hard to accomplish. Any insufficiency or errors in this paper are of course of my own.

\section{References}

Alexander Clark and Rémi Eyraud. 2007. Polynomial identification in the limit of substitutable context-free languages. Journal of Machine Learning Research, 8:1725-1745.

Alexander Clark and Ryo Yoshinaka. 2014. Distributional learning of parallel multiple context-free grammars. Machine Learning, 96(1-2):5-31.

Alexander Clark, Rémi Eyraud, and Amaury Habrard. 2009. A note on contextual binary feature grammars. In EACL 2009 workshop on Computational Linguistic Aspects of Grammatical Inference, pp. 33-40.

Alexander Clark. 2010a. Distributional learning of some context-free languages with a minimally adequate teacher. In J. Sempere and P. García, editors, ICGI, LNCS 6339, pp. 24-37. Springer.

Alexander Clark. 2010b. Learning context free grammars with the syntactic concept lattice. In J. Sempere and P. García, editors, ICGI, LNCS 6339, pp. 38-51. Springer.
Alexander Clark. 2010c. Towards general algorithms for grammatical inference. In M. Hutter, F. Stephan, V. Vovk, and T. Zeugmann, editors, ALT, LNCS 6331, pp. 11-30. Springer.

Yuichi Kaji, Ryuichi Nakanishi, Hiroyuki Seki, and Tadao Kasami. 1992. The universal recognition problems for parallel multiple context-free grammars and for their subclasses. IEICE Transaction on Information and Systems, E75-D(7):499-508.

Makoto Kanazawa and Ryo Yoshinaka. 2015. Distributional learning and context/substructure enumerability in non-linear tree grammars. In Formal Grammar 20th International Conference, FG 2015, Barcelona, Spain, August 8-9, 2015. Proceedings. to appear.

Anna Kasprzik and Ryo Yoshinaka. 2011. Distributional learning of simple context-free tree grammars. In J. Kivinen, C. Szepesvári, E. Ukkonen, and T. Zeugmann, editors, ALT, LNCS 6925, pp. 398-412. Springer.

Alexander Okhotin. 2001. Conjunctive grammars. Journal of Automata, Languages and Combinatorics, 6(4):519-535.

Hiroyuki Seki, Takashi Matsumura, Mamoru Fujii, and Tadao Kasami. 1991. On multiple context-free grammars. Theoretical Computer Science, 88(2):191-229.

Hiromi Shirakawa and Takashi Yokomori. 1993. Polynomial-time MAT learning of c-deterministic context-free grammars. Transaction of Information Processing Society of Japan, 34:380-390.

Ryo Yoshinaka and Makoto Kanazawa. 2011. Distributional learning of abstract categorial grammars. In S. Pogodalla and J.-P. Prost, editors, LACL, LNCS 6736, pp. 251-266. Springer.

Ryo Yoshinaka. 2008. Identification in the limit of $k, l$-substitutable context-free languages. In A. Clark, F. Coste, and L. Miclet, editors, ICGI, LNCS 5278, pp. 266-279. Springer.

Ryo Yoshinaka. 2011a. Efficient learning of multiple context-free languages with multidimensional substitutability from positive data. Theoretical Computer Science, 412(19):1821-1831.

Ryo Yoshinaka. 2011b. Towards dual approaches for learning context-free grammars based on syntactic concept lattices. In G. Mauri and A. Leporati, editors, DLT, LNCS 6795, pp. 429-440. Springer.

Ryo Yoshinaka. 2012. Integration of the dual approaches in the distributional learning of context-free grammars. In A. H. Dediu and C. Martín-Vide, editors, LATA, LNCS 7183, pp. 538-550. Springer.

Ryo Yoshinaka. 2015. Learning conjunctive grammars and contextual binary feature grammars. In A. H. Dediu, E. Formenti, C. Martín-Vide, and B. Truthe, editors, LATA, LNCS 8977, pp. 623-635. Springer. 